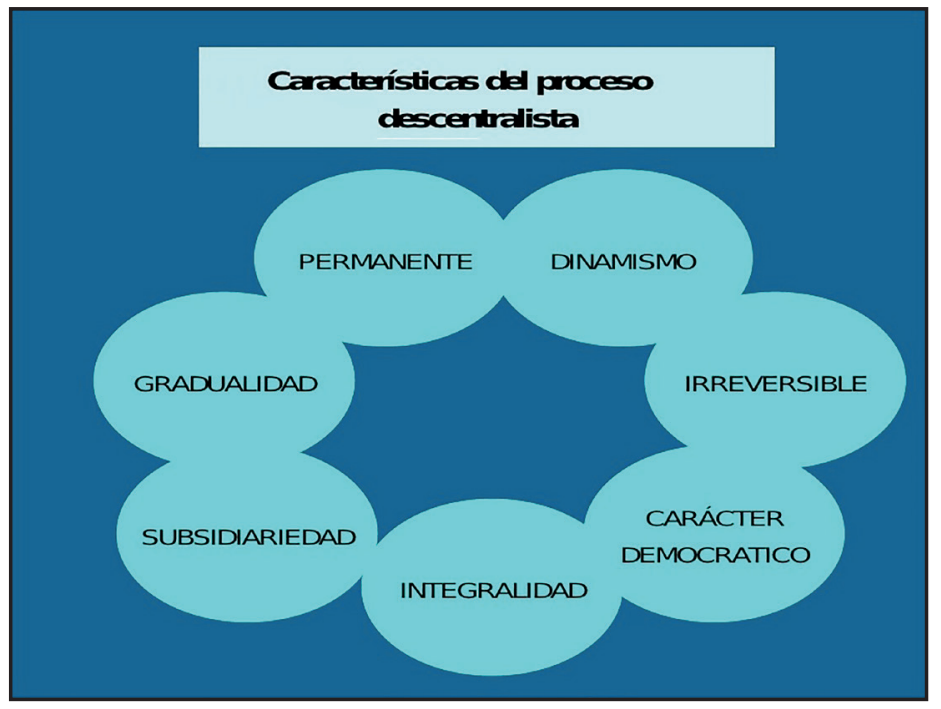

https://www.google.com.ec/url?sa=i\&rct=j\&q=\&esrc=s\&source=images\&cd=\& cad=rja\&uact=8\&ved=0ahUKEwinw6P4mYHTAhVhrVQKHTmtBi0QjRwIBw\&u rl=https $\% 3 \mathrm{~A} \% 2 \mathrm{~F} \% 2 \mathrm{Fes}$.slideshare.net $\% 2 \mathrm{Flitzy} 26 \% 2 \mathrm{Fla}$-descentralizacion-yregionalizacion-en-el-peru\&bvm=bv.151426398,bs.2,d.eWE\&psig=AFQjCNEy WreieVc24FAX6JNwoqbhsxJogg\&ust=1491064899388809

\title{
DESCENTRALIZACIÓN Y DEMOCRACIA EN EL ECUADOR. BREVES APUNTES PARA LA INVESTIGACIÓN Pablo Ortiz-T. ${ }^{1}$
}

Correspondencia: portiz@ups.edu.ec

1 PhD y Msc. en Ciencias Políticas y Sociólogo. Director de la Carrera de Gestión de Desarrollo Local de la Universidad Politécnica Salesiana (UPS) y Coordinador del Grupo de Investigación sobre Estado y Desarrollo GIEDE. Contacto: portiz@ups.edu.ec 


\title{
Resumen
}

La correlación entre descentralización y democracia está mediada de múltiples factores, entre los cuales se resalta el carácter y papel del Estado, la articulación de éste con los procesos de globalización, el tipo de organización territorial que se impulsa, cambia o reproduce, y la correlación de fuerzas existente en el escenario de la lucha política. El texto pasa revista, de manera breve, a estos aspectos, tomando como referencia el proceso ecuatoriano de las últimas dos décadas, cuyos resultados los alude sin dejar de lado en el apunte central una perspectiva panorámica que abre nuevas interrogantes de cara a definir nuevas líneas de producción de conocimientos y de debate.

Palabras clave: descentralización, Estado, democracia, participación, desarrollo local, organización territorial, identidad cultural, formación capitalista.

\section{DECENTRALIZATION AND DEMOCRACY IN ECUADOR; BRIEF NOTES FOR RESEARCH}

\begin{abstract}
The correlation between decentralization and democracy is mediated by a number of factors, including the character and role of the State, its connection with globalization processes, the type of territorial organization that is promoted, changed or reproduced, and the correlation of forces that exist in the political arena. The text briefly reviews these aspects, taking as reference the Ecuadorian process of the last two decades, whose results are mentioned without disregarding a panoramic perspective that opens new questions in order to define new production lines of knowledge and debate.
\end{abstract}

Keywords: decentralization, State, democracy, participation, local development, territorial organization, cultural identity, capitalist formation. 


\section{Apunte uno}

Una rápida mirada a la cartografía histórica del Ecuador, desde 1830 hasta la actualidad, permite advertir que la malla político-administrativa del país conserva una huella colonial y muy tenuemente un rezago anterior al siglo XVI dentro de los antiguos cacicazgos como lo ha explicado Frank Salomon (1986). También se refleja la impronta que ha dejado en el espacio el tipo de capitalismo dependiente y primario exportador implantado. ¿Qué ha cambiado en esos procesos de configuración político-territorial en el Ecuador actual? ¿Acaso una mayor descentralización implica más democracia y desarrollo? ¿Cuáles son esas interrelaciones?

Para esbozar una aproximación preliminar a estas interrogantes, es importante referir de forma breve y retrospectiva lo que ha sido el caso ecuatoriano. $Y$ es que la organización político-territorial de un país forma parte de la configuración de su formación histórico-social, lo que remite a entender la correlación directa entre un tipo de territorialidad vigente en un determinado momento, un tipo de Estado y un determinado orden económico-social. A propósito de eso resulta pertinente traer a colación la ya clásica premisa marxista en torno al Estado, sus instituciones y sus reglas: «...ni las relaciones jurídicas como las formas del Estado pueden comprenderse ni por sí mismas ni por la así llamada evolución general del espíritu humano, sino que tienen sus raíces, más bien, en las relaciones materiales de la existencia" (Marx, 1974, pág. 76). Relaciones de existencia que remiten a los procesos de producción e intercambio de bienes y de personas. De ahí el desafío de comprender la interrelación entre las 
dinámicas territoriales, la formación capitalista y el sistema político. A una estructura social capitalista se corresponde una estructura espacial concreta y las relaciones sociales y económicas específicas tienen su correlato o dimensión espacial. El espacio social debe ser entendido como la articulación de las relaciones sociales y económicas, y la producción del espacio social no es sino la estructuración del espacio en el marco de un determinado modo de producción (Lipietz, 1979; Harvey, 2001).

Si en el contexto de la inserción del país al mercado mundial, entre finales del siglo XIX hasta mediados del siglo $\mathrm{XX}$ a través de la agro-exportación cacaotera o bananera, se afianzó un tipo de Estado oligárquico y de poder burgués agroexportador, comercial y bancario, a la par que un orden territorial altamente desigual, a partir de la segunda mitad del siglo XX se reafirma la crisis de dicho Estado oligárquico, la emergencia de otras fracciones burguesas más modernas ligadas con la agroindustria, la manufactura industrial y las finanzas, junto con una mayor fragmentación político-administrativa, que deja inalteradas las asimetrías territoriales y la vigencia de un régimen político altamente excluyente. Entre 1897 y 1958, año en que el Ministerio de Economía publica una actualización de la Ley de División Territorial, de los 53 cantones existentes en el inicio de ese período se incrementan a 95; significa que en 61 años se crearon 42 cantones (IGM, 2011, pág. 29).

Aquella crisis del Estado oligárquico y su correlato territorial dio paso a la modernización capitalista y a la reestructuración político-administrativa bajo la dirección y/o tutela militar en los sesenta y setenta, que enfatizó la planificación estratégica central y planificación sectorial y 
regional, con una perspectiva monocromática y positivista del cambio²: de la sociedad tradicional a la moderna, de la vieja hacienda a la empresa agrícola, de la sociedad rural a la urbana, de campesinos a proletarios, de artesanos a industriales. En suma, un tipo de desarrollismo, entendido como crecimiento y acumulación de capital a secas, ligado con la consolidación del proyecto de Estado-nación.

Sin embargo, en la transición del Estado desarrollista al Estado neoliberal, entre 1981 y 1993 se crearon 67 nuevos cantones, "...haciendo tabla rasa de la Ley de Régimen Seccional que exigía, en ese entonces, para que una cabecera parroquial adquiera la categoría de cabecera cantonal debe tener 10 mil o más habitantes; no obstante, se elevaron a la categoría de cabecera cantonal a cabeceras parroquiales con hasta apenas 500 habitantes" (IGM, 2011, pág. 29). Según Pierre Gondard, "...en los datos del Censo de Población de 1974 constan 114 cantones y 714 parroquias rurales y en el de 2001 más de 200 cantones y cerca de 2.000 parroquias rurales. El caserío quiere ser parroquia y la parroquia cantón lo que logran a veces fuera de toda justificación técnica, sin más relación con la población atendida, llegando a algunas

2 Me refiero al itinerario de la planificación en el Ecuador, que arranca en 1948 cuando el Ministerio de Economía proyectó un "Plan de Fomento a la Producción", y en 1954 se crea la Junta Nacional de Planificación y Coordinación Económica (JUNAPLA), que durante treinta años elaboró los denominados Planes de Desarrollo, hasta cuando en 1978 se crea -junto con la nueva Constitución- el Consejo Nacional de Desarrollo CONADE, que sería la antesala de las otras dos instancias de planificación central en 1998 y 2008, el Consejo Nacional de Modernización (CONAM) y la Secretaría Nacional de Planificación y Desarrollo (SENPLADES) respectivamente. 
incongruencias y desigualdades a veces abismales" (Gondard, 2005, pág. 55).

En alguna medida el modelo neoliberal impuso de forma simultánea, por un lado, la re-primarización de la economía y la desarticulación del Estado central, y por otro lado, un tipo de organización territorial y descentralización que fuera funcional con las necesidades del capital ligado al mercado externo y de los sectores hegemónicos beneficiarios de dicho modelo (Ramón Valarezo, 2004).

En otras palabras el proceso de dominación política que consolida un tipo de capitalismo dependiente y primarioexportador requiere de un modelo territorial excluyente, estratificado e inequitativo, útil a las necesidades del capital, de sobre-explotación de mano de obra y de la naturaleza, todo lo cual se expresa en Iña dirección de las políticas de inversiones públicas en infraestructura vial o de telecomunicaciones, que priorizaron únicamente zonas extractivas orientadas hacia los grandes mercados mundiales. En ese marco las demandas de áreas periféricas o las necesidades existentes de establecer o consolidar redes internas de movilidad a lo ancho y largo del país no son prioritarias, todo lo cual ahonda un esquema de crecimiento desigual de las regiones, consolidando a finales del siglo XX -como lo anota Gondard- el bicentralismo de Quito y Guayaquil y un modelo degenerativo de asimetría en los territorios (Gondard, 2005, pág. 57).

Tal proceso de organización asimétrica del territorio va acompañado de la dinámica demográfica: la población urbana del país pasó entre 1778 y 1950 del $15 \%$ al $28 \%$, mientras que en el 2010 llega al 62,8\%. Lo propio pasó con el número de localidades de más de 10 mil habitantes, que 
pasaron de 13 en 1950 a 109 en 2010, como se aprecia en la tabla 1.

Sin duda, la red de centros poblados existentes y su distribución están asociados al modelo de reproducción desigual del desarrollo regional y de la formación capitalista impuesta (Ibarra, 2005; Ramón Valarezo, 2004). Es decir, establecer a unas zonas como reservas de mano de obra no calificada o de fuerza de trabajo de bajo valor, definir a otras regiones que concentran acumulación de capital y una mayor densidad de mano de obra calificada y ciertas ramas de la producción, y finalmente consolidar un tercer tipo de zona de aquellos sectores directamente articulados con los centros de negocios e inversión de capital con fuerza de trabajo de mayor valor y altamente calificada.

\begin{tabular}{|l|c|c|c|c|c|c|c|}
\hline \multicolumn{7}{|c|}{ Tabla 1 } \\
\hline \multicolumn{7}{|c|}{ Localidades de +10 mil habitantes, según región y censo } \\
\hline Regiones & 1950 & 1962 & 1974 & 1982 & 1990 & 2001 & 2010 \\
\hline Costa & 5 & 13 & 20 & 30 & 38 & 46 & 57 \\
\hline Sierra & 8 & 8 & 15 & 19 & 25 & 31 & 43 \\
\hline Amazonía & 0 & 0 & 0 & 0 & 2 & 7 & 8 \\
\hline Galápagos & 0 & 0 & 0 & 0 & 0 & 0 & 1 \\
\hline Subtotal & 13 & 21 & 35 & 49 & 65 & 84 & 109 \\
\hline Fuente: INEC, Censos Nacionales, varios años \\
\hline Elaboración: IGM-Ministerio de Defensa-Senplades, 2011 \\
\hline
\end{tabular}

En ese contexto, los asentamientos humanos responden a los modelos de desarrollo impuestos a lo largo del devenir histórico, en función de las diferentes formas de apropiación de la riqueza. El auge cacaotero 
desarrolló algunas ciudades en el interior de la costa (Chiriboga, 1980; Deler, 1994). Posteriormente la época bananera desarrolló puertos y accesos a ellos y los años del desarrollismo y de la sustitución de importaciones e industrialización impulsaron la red vial entre Quito y Cuenca y buena parte de la sierra centro-norte y para finales de los sesenta y los años setenta, el "boom petrolero" incorpora a la Amazonía nor-oriental a través de la red de oleoductos y facilidades de la industria petrolera. Esta dinámica en buena medida ha marcado la pauta del poblamiento del territorio y de una estructura nacional desigual y poco uniforme (Deler, 2007).

Esta estructura territorial asimétrica expresa en buena medida el tipo de intervención que el Estado ha realizado a lo largo de este proceso, donde la intensidad de las inversiones realizadas en infraestructura (redes eléctricas, carreteras, puertos, aeropuertos, oleoductos, redes de telecomunicación, etc.) llega a modificar en esos casos el territorio, paliando en la mayoría de situaciones la incapacidad de la iniciativa privada para suscitar el desarrollo local.

Adicionalmente esta estructura asimétrica es uno de los principales obstáculos al proceso de descentralización, no solo en el Ecuador, sino en varios países de América Latina. "En la mayoría de gobiernos locales, el personal técnico o profesional es muy reducido, la información llega tarde, la gestión es difícil y muchas funciones no pueden cumplirse y, sobre todo, muchos servicios no pueden darse a la población (....) Esta -digamos- "dominante urbana" en la cultura de quienes legislan y quienes deciden sobre políticas públicas, que erróneamente, además se asocia con "desarrollo" y "progreso", en tanto lo rural se imagina 
siempre "tradicional", o "estancado" hace que no se diseñen servicios para condiciones de ruralidad ni para pequeños centros urbanos". (Grompone, Hernández, \& Huber, 2008, pág. 10). En la misma dirección José Arocena apunta: "En América Latina más allá de las diferencias entre los distintos países, numerosos indicadores están mostrando la presencia de instituciones débiles y con poca capacidad de incidencia en la vida de la comunidad. Esta rápida constatación de la debilidad de las instituciones locales latinoamericanas admite evidentemente excepciones" (Arocena, 1997, pág. 44).

La debilidad de los llamados gobiernos autónomos descentralizados (GAD) en el caso del Ecuador, para alcanzar autonomía fiscal o desarrollar capacidad de tributación en sus jurisdicciones, limita severamente las posibilidades de que puedan asumir competencias y dirigir procesos de desarrollo local.

\section{Apunte 2}

La noción moderna o dominante de democracia está ligada a la expansión capitalista y al proyecto de Estadonación. Los actuales procesos de globalización, sin duda, impactaron relativamente en un desplazamiento relativo de los Estados centrales y su rol, más allá de los mitos neoliberales sobre el papel del Estado en el capitalismo, como aquel que considera que puede haber un desarrollo espontáneo del mercado sin interferencia del Estado y el que plantea la disminución del papel del Estado en la sociedad.

Sobre lo primero, las experiencias históricas del desarrollo del capitalismo europeo, de los Estados Unidos, 
de Japón y de otros países asiáticos muestran la falsedad del discurso neoliberal: el laissez faire ha existido por excepción y no como regla en cada uno de esos casos (Lander, 2002).

Sobre lo segundo, se ha enfatizado en la disminución de la soberanía de los Estados frente a las fuerzas transnacionales del mercado: "probablemente es más apropiado decir que las funciones principales de los Estados-nación -la regulación de la circulación monetaria, de los flujos económicos, los movimientos migratorios, las normas culturales, los valores culturales, etc.- han mantenido su importancia, pero se han transformado debido a los procesos contemporáneos de globalización. El radical cambio cualitativo se reconoce sobre todo en términos de soberanía. Los Estados-nación ya no pueden pretender el papel soberano de autoridad máxima, como en la era moderna" (Negri, 2004, pág. 95) Tesis que Edgardo Lander acota y puntualiza:

"El discurso de la reducción del peso o la intervención del Estado, o en torno a la impotencia de éste ante las fuerzas globales del mercado, ha sido más un recurso ideológico destinado a la contención de las demandas democráticas y al debilitamiento relativo de los Estados del sur frente al capital transnacional, que una tendencia general que afecte en forma similar a todos los Estados del mundo. Divergen radicalmente las transformaciones principales de la mayor parte de los Estados periféricos, por un lado, y las de los Estados centrales por el otro, en particular en el caso del principal Estado-nación del sistema del mundo actual: Estados Unidos" (Lander, 2002, pág. 67).

En ese contexto, la descentralización debe entenderse como parte de las reformas estatales, en tanto responden 88 
a problemas de su estructura y funcionamiento y están relacionadas con la distribución de recursos, con la asignación de atribuciones y potestades, y más aún si se entiende al Estado como una construcción histórica y una forma de organización social y política soberana y coercitiva, que tiene el poder de regular la vida nacional dentro de un territorio determinado (Arocena, 1997; Sánchez, 2004; Ojeda, 2004). Desde una perspectiva liberal clásica (como la de Adam Smith), se entendería al Estado como un "vigilante nocturno" minimalista, que proporciona la mejor cobertura para el crecimiento económico. O si se lo entiende como un sistema de instituciones relativamente autónomo, cuya función central está en asegurar la reproducción del orden vigente y procesar prioritariamente las demandas de las clases dominantes en la sociedad. Para ello requiere afianzar su legitimidad y lo hace a través de la función de constituirse en factor de cohesión de los distintos niveles de una formación social y de regulación de su equilibrio global, en cuanto sistema (Bobbio, 2014; Bobbio, Matteucci, \& Pasquino, 1991; Habermas, 1998). Más crítica e integral, una perspectiva marxista remite a una noción del Estado como portador de los intereses de las clases dominantes, algo que no opera mecánica ni linealmente; en ese sentido, nunca puede entenderse al Estado como una fuerza neutral que represente un interés general, pues las reglas que genera ese Estado sirven en lo fundamental a la reproducción del orden capitalista y por ende a los intereses de la clase dominante o a determinadas fracciones de ella o a un bloque de poder, según su propia configuración. Aquello está distante de la premisa que plantee que el Estado pueda representar o acoger los intereses de todo un pueblo o de los sectores subalternos. ${ }^{3}$

3 Es una expresión que refiere a Marx en su famoso "Manifiesto Comunista" de 1848. 
El propio Marx (1971) en "El 18 Brumario de Luis Bonaparte" sugiere que el Estado podría alcanzar una autonomía relativa logrando un equilibrio entre sectores rivales de la clase dominante, e incluso permitirle imponer medidas de bienestar que beneficien a largo plazo a los intereses del capital, pero de forma simultánea garantizar estabilidad para crear una mano de obra cualificada y productiva. Esta última premisa llevaría a pensar en el Estado como una arena en disputa, un espacio de confrontación, cuya orientación dependerá de la correlación de fuerzas.

A partir de esos y otros elementos conceptuales, la descentralización puede ser abordada de varias maneras, dependiendo de la perspectiva teórica y las opciones políticas. Puede ser entendida desde una perspectiva funcionalista e institucional como un instrumento del propio Estado para fortalecerse y optimizar su rol, incrementar su capacidad de penetrar en la sociedad y de controlarla mejor, pero también de prestar mejores servicios y procesar más eficazmente algunas de las demandas sociales; o también desde una óptica neoliberal la descentralización puede ser parte importante de la desregulación del Estado y de la economía, de la reducción del tamaño del aparato estatal y del traspaso total de varias de sus funciones y competencias a entes privados (fundaciones, corporaciones, iglesias o empresas), en cuyo caso, la descentralización adquiere un sesgo privatizador y funcional a los intereses del mercado y del capital como eje organizador de la vida social (Lander, 2002; Chiriboga, 2009). De forma irónica se podría decir que la "fortaleza" del Estado está en su propia debilidad, pues es él mismo quien crea condiciones para la desregulación y la privatización, desplazándolo a un segundo plano, como ha sucedido en el marco de la política neoliberal en varios países del mundo (Saad-Filho, 2005). 
Sin embargo como antítesis y respuesta a esa dinámica hegemónica, en el marco de la globalización y la dinámica del capital (Gallicchio, Camejo, \& Marchesi, 2005; Harvey, 2001) también deben entenderse la emergencia de los movimientos populares y sus demandas de transformación del Estado y del orden social, reivindicando su propia capacidad de formular otro tipo de propuestas políticas o proyectos políticos, pasando por reinvidicar derechos individuales, sociales o colectivos, hasta el reconocimiento de su identidad y el derecho a la diferencia, como lo anota Arturo Escobar: "...el punto consiste en distinguir aquellas formas de globalización de lo local que pueden convertirse en fuerzas políticas efectivas para la defensa del lugar y de las identidades basadas-en-lugar, así como aquellas formas de localización de lo global que desde lo local, se pueden llegar a utilizar para su propia ventaja (...) para construir el lugar como un proyecto, para hacer de los imaginarios basados-en-lugar en un crítica radical al poder, y para alinear la teoría social como una crítica al poder en favor del lugar, se requiere que nos aventuremos en otros terrenos". (Escobar, 2012, pág. 126).

Y es que la identidad local se construye sobre la base de dos dimensiones: la histórica y la territorial. La primera alude a la memoria viva de un grupo humano que se reconoce en las huellas de un pasado y representa continuidad y ruptura entre el pasado, el presente y el proyecto. El segundo, es el espacio significativo para el grupo que lo habita, que genera una relación desarrollada en un nivel profundo de conciencia. Representa permanencia y ausencia, continuidad y ruptura. El proceso de construcción de identidad, según Gallicchio y otros, "...se produce en un sistema de relaciones (la dimensión de la relación con otros es muy importante); se apoya en la idea de unidad de sí 
mismo a través de cierto lapso de tiempo (permanencia); se apoya también en la idea de diferencia (uno es uno mismo y no otro); permite la existencia de límites (como fronteras, no como cierres) que habilitan intercambios selectivos con otros; y se afirma en la capacidad de rememorar lo que se vivió y se es, y adecuarlo a los nuevos contextos, generando capacidad de reconstruir la identidad" (Gallicchio, Camejo, \& Marchesi, 2005, pág. 57)

Para Di Pietro, la "identidad colectiva local es producto de una continuidad en el tiempo vivida conscientemente por un grupo humano, generadora de una acumulación cultural en términos de sistema de normas y valores. Toda sociedad es un sistema de valores interiorizado por sus miembros. Implica la pertenencia a una comunidad y reconocerse en una identidad colectiva compartida" (Di Pietro Paolo, 2003, pág. 26)

Aquello no es sino remitirse a la esfera del interés común y de lo público. En la perspectiva de Habermas, el concepto de esfera pública presupone igualdad de derechos individuales (sociales, políticos y civiles) y discusión, sin violencia o cualquier otro tipo de coacción, de problemas mediante la autoridad negociada. La esfera pública, por lo tanto, es el espacio intersubjetivo, comunicativo, en el cual las personas o colectivos tematizan sus inquietudes por medio del entendimiento mutuo (Habermas, 1998, pág. 92).

Un ejemplo de aquello se encuentra en la incursión de líderes o lideresas indígenas en la sierra ecuatoriana como autoridades locales en los años noventa. Autores como John Cameron (2005), Víctor Hugo Torres (2004), Rosales, Puebla, \& Velasco (1988) y (Sánchez, 2004) refieren 
ampliamente estas experiencias y llaman la atención sobre las formas en que el diseño de las instituciones municipales y las estrategias de liderazgo existentes han tomado forma y han sido moldeadas al calor de las relaciones de poder a nivel local. Según Cameron, “...una de las funciones más importantes de los líderes (locales) fue realizar esfuerzos de democratización mediante el impulso de coaliciones locales, a través de negociaciones entre clases y entre etnias. Esas experiencias muestran que el éxito de instituciones creadas para profundizar la participación popular en el gobierno local está condicionada por el grado en que su diseño refleja relaciones sociales de poder local" (Cameron, 2005, pág. 367).

En la misma línea reflexiva, Tenório y Monje-Reyes resaltan que "el fomento de las prácticas participativas es un elemento que impacta positivamente en el empoderamiento de la población respecto del devenir de su comunidad. Uno de los elementos hallados que apunta hacia este fomento es la apertura de espacios públicos. Al abrirse estos espacios, se abre también la posibilidad de que se instalen prácticas participativas que tengan impacto en la generación de políticas públicas. De esta manera, estas acciones propenden a la generación de una nueva calidad de personas, en la que se asienta la idea del poblador como creador de derechos y cumplidor de deberes, con lo cual se rompe la dinámica predominante de carácter netamente asistencialista. Además, la capacidad de organización que permiten la inclusión social, cuestión que contribuye directamente al desarrollo local" (Tenório \& Monje-Reyes, 2010, pág. 102)

El proceso contemporáneo de descentralización aún enfrenta retos y tareas pendientes, a pesar de las 
estrategias y avances existentes (Barrera Guarderas, 2016; SENPLADES, 2011; Vázquez, 2015). El reconocimiento de procesos participativos y prácticas deliberativas, incluso a nivel de pautas y normas establecidas, es un avance, aunque es evidente el aún débil tejido social y apropiación tanto de parte de la institucional local, como de parte de las organizaciones sociales y los llamados sectores ciudadanos. Prevalecen las lógicas y racionalidades del aparato burocrático por sobre las prácticas deliberativas y de planificación realmente existentes dentro de la propia sociedad civil (Vivanco Cruz, 2016; Ruiz Giraldo, 2013).

Si el proceso neoliberal de descentralización acentuó las asimetrías territoriales, resta por comprender mejor y más profundamente los alcances de la nueva etapa iniciada a raíz de la Constituyente de 2008 (Rast, 2008). Aún está pendiente viabilizar el futuro de ciertas localidades, que pasa por integrarlas a espacios más agregados, de forma paralela a profundizar mayores reformas políticas que interpelen y superen las viejas prácticas clientelares y un tipo de cultura política tradicional donde aún pesan los caudillismos. Hay una descentralización política que debe revertir las condiciones estructurales de inequidad y asimetría territorial, con condiciones acopladas a los diversos grados de desarrollo local territorial, y a las capacidades de los distintos gobiernos locales. Como lo señalan Vázquez (2015) y Barrera Guarderas (2016), a pesar de la bonanza fiscal que incrementó las rentas de los GAD, aquello no se tradujo en mayor institucionalidad, autonomía en la gestión gubernativa, sino que ahondó la dependencia, el fraccionamiento de los poderes locales y hasta incrementó el intervencionismo del gobierno central, que invadió competencias para atender o procesar demandas locales desatendidas, dejando diferido el 
proceso de traspaso de competencias y una renovación de la democracia local.

\section{Bibliografía citada}

Arocena, J. (1997). Arocena, J. (1997). El desarrollo local frente a la globalización. Hacia un nuevo modelo de gestión local, Municipio y Sociedad Civil en Argentina, 43-58. Municipio y Sociedad Civil en Argentina, 43-58.

Bachrach, P. (1973). Crítica de la Teoría Elitista de la Democracia. Buenos Aires: Amorrortu.

Barrera Guarderas, A. (2016). El Proceso de Descentralización en Ecuador: 8 Años después de Montecristi. Flacso Centro de Investigaciones de Políticas Públicas y Territorio CITE. Quito: Friedrich Ebert Stiftung ILDIS FLACSO Cite.

Bobbio, N. (2014). Estado, Gobierno y Sociedad. Por una Teoría General de la Política. México DF.: Fondo de Cultura Económica FCE.

Bobbio, N., Matteucci, N., \& Pasquino, G. (1991). Diccionario de Política. México DF.: Siglo XXI Editores.

Cameron, J. D. (2005). Municipal Democratisation in Rural Latin America: Methodological insights from Ecuador. (C. Palmer, Ed.) Bulletin of Latin American Research, 24(3), 367-390.

Chiriboga, M. (1980). Jornaleros y gran propietarios en 135 años de exportación cacaotera, 1790-1925. Quito: CIESE. 
Chiriboga, M. (2009). Competitividad Territorial y el Rol de los Gobiernos Autónomos Descentralizados. CONGOPE, Dirección de Desarrollo Económico y Competitividad Territorial (DIDECT). Quito: CONGOPE.

Dahl, R. (2000). La Democracia y sus Críticos. Barcelona: Paidós.

Deler, J. P. (1994). Transformaciones regionales y organización del espacio ecuatoriano entre 1830 y 1930 . En J. Maiguashca, Historia y región en el Ecuador: 1830-1930 (págs. 295-354). Quito: Corporación Editora Nacional.

Deler, J. P. (2007). Ecuador del espacio al estado nacional. Quito: Universidad Andina Simón Bolívar-Instituto Francés de Estudios Andinos IFEA.

Di Pietro Paolo, L. J. (2003). Hacia un Desarrollo Integrador y Equitativo: una Introducción al Desarrollo Local. En D. y. Burin, Desarrollo Local. Una Respuesta a Escala Humana a la Globalización (págs. 13-50). Buenos Aires: CICCUS-La Crujía.

Dussel, E. (2006). 20 Tesis de Política. México DF.: Siglo XXI.

Escobar, A. (2012). Una Minga para el Postdesarrollo. Lugar, Medio Ambiente y Movimientos Sociales en las Transformaciones Globales. Bogotá: Desde Abajo.

Gallicchio, E., Camejo, A., \& Marchesi, A. (2005). Desarrollo local y descentralización en América Latina: nuevas alternativas de desarrollo. Montevideo: CLAEH-DIBA.

Gondard, P. (2005). Ensayo en torno a las regiones de Ecuador Herencias y reestructuraciones. (F. Rivera, Ed.) Ecuador Debate(66), 45-60. 
Grompone, R., Hernández, R., \& Huber, L. (2008). Ejercio de Gobierno Local en los Ámbitos Rurales. Presupuesto, Desarrollo e Identidad. Lima: Instituto de Estudios Peruanos IEP.

Habermas, J. (1998). Facticidad y Validez. Sobre el Derecho y el Estado Democrático de Derecho en Términos de Teoría del Discurso. Madrid: Trotta.

Habermas, J. (Abril de 2005). Tres modelos de democracia. Sobre el concepto de una política deliberativa. (C. d. (CISPO), Ed.) POLIS Revista Latinoamericana(10), 1-10.

Harvey, D. (2001). Spaces of Capital. Toward a Critical Geography. New York: Routledge, Edinburgh University Press.

Ibarra, H. (Agosto de 2005). Acerca del Localismo Ecuatoriano.

(F. R. Vélez, Ed.) Ecuador Debate(65), 53-66.

IGM. (2011). Atlas de la República del Ecuador. Quito: Instituto Geográfico Militar-Senplades-Ministerio de Defensa.

Lander, E. (mayo-agosto de 2002). La Utopía del Mercado Total y el Poder Imperial. (M. L. Maya, Ed.) Revista Venezolana de Economía y Ciencias Sociales, 8(2), 51-79. Obtenido de http://www.ucv.ve/fileadmin/user_ upload/faces/problemas_sociales_contemporaneos/ CESOC/MAYO_AGOSTO_2_2002_LA_REFORMA_ PETROLERA_EN_VENEZUELA.pdf

Lipietz, A. (1979). El Capital y su Espacio. México DF.: Siglo $\mathrm{XXI}$.

Marx, K. (1971). El 18 Brumario de Luis Bonaparte. Barcelona: Ariel. 
Marx, K. (1974). Elementos fundamentales para la Crítica de la Economía Política (borrador) 1857-1858. Córdoba Pasado y Presente: Pasado y Presente.

Negri, A. (2004). Guías. Cinco Lecciones en torno al Imperio. Barcelona: Paidós.

Ojeda, L. (abril de 2004). ¿Por qué la Descentralización No Avanza? (F. Rivera, Ed.) Ecuador Debate(61), 95-116.

Pauta Calle, F. (2013). Ordenación Territorial y Urbanística: un Camino para su Aplicación en el Ecuador. Cuenca: Universidad de Cuenca.

Ramón Valarezo, G. (2004). El Estado y la Cuestión Local en el Ecuador Contemporáneo. En G. y. Ramón, El Desarrollo Local en el Ecuador: Historia, Actores y Métodos (págs. 77-117). Quito: Comunidec-Abya Yala.

Rast, L. \&. (2008). Buenas Prácticas en Participación. Quito: DED-Ceplaes.

Rosales, M., Puebla, J., \& Velasco, M. (1988). El Desafío Local: El Municipio como Agente de Desarrollo. Quito: El Conejo.

Ruiz Giraldo, C. (2013). La Transición hacia una Nueva Forma de Descentralización: El Proceso de Reforma del Estado en Ecuador a raíz de la Aprobación de la Constitución del 2008. En A. y. Krainer, Actores, Procesos y Retos de la Descentralización en Ecuador. Una Mirada Retrospectiva a la Década 1998-2008 (págs. 95-136). Quito: FLACSO.

Saad-Filho, A. a. (2005). Neoliberalism: A critical reader. Chicago: Chicago University of Chicago Press. 
Salomon, F. (1986). Salomon, Frank Native lords of Quito in the age of the Incas: The political economy of north Andean chiefdoms. New York: Cambridge University Press.

Sánchez, J. (abril de 2004). Descentralización, Macroeconomía y Desarrollo Local. (F. Rivera, Ed.) Ecuador Debate(61), 77-94.

SENPLADES. (2011). Lineamientos Generales para la Planificación Territorial Descentralizada. Quito: SENPLADES.

SENPLADES. (2013). Plan Nacional de Desarrollo/Plan Nacional para el Buen Vivir 2013-2017. Quito: Secretaría Nacional de Planificación y Desarrollo SENPLADES.

Tenório, F. G., \& Monje-Reyes, P. (2010). Ciudadanía, Participación y Desarrollo Local. Santiago de Chile: ARCIS.

Torres, V. H. (2004). El Mejoramiento de la Vida también es cuestión de Métodos: un Recuento de la Metodología Participativa y el Desarrollo Local. En G. y. Ramón, El Desarrollo Local en el Ecuador: Historia, Actores y Métodos (págs. 171-227). Quito: Comunidec-Abya Yala.

Vázquez, M. C. (2015). La Descentralización: Un Camino para el Desarrollo Territorial. En D. \&. Martínez Godoy, Desarrollo Territorial en Ecuador. Situación Actual y Perspectivas (págs. 61-76). Quito: CONGOPE-Abya Yala.

Vega Jiménez, N. (2008). Principales Factores de la Descentralización en el Ecuador . En Y. Melgar Hermoza, Procesos de Descentralización en la Región Andina (págs. 93-125). Lima: Internationale Weiterbildung und Entwicklung gGmbH Inwent. 
Vivanco Cruz, L. (2016). La Participación Ciudadana: Una Visión Sistemática en el Marco del Ordenamiento Territorial en el Ecuador. Cuenca: Universidad de Cuenca. 